\title{
Beyond the time independent mean field theory for nuclear and atomic reactions: Inclusion of particle-hole correlations in a generalized random phase approximation
}

\author{
J.C. Lemm \\ Institut für Theoretische Physik, Universtät Münster, 48149 Münster, Germany \\ B.G. Giraud \\ Service de Physique Théorique, DSM-CEA Saclay, 91192 Gif/Yvette, France
}

A. Weigunyt

Yukawa Institute for Theoretical Physics, Kyoto University, Kyoto 606, Japan

(November 21, 2018)

\begin{abstract}
The time independent mean field method (TIMF) for scattering defines biorthonormal sets of singleparticle wave functions and corresponding creation and annihilation operators. $2 p-2 h$ correlations can be introduced through a generalized random phase approximation; $1 p-1 h$ contributions vanish (Brillouin theorem). While the general variational method for scattering by Giraud and Nagarajan solves inhomogeneous Euler equations by inversion of the standard, hermitean Hamiltonian, the present approach diagonalizes a non-hermitean Hamiltonian, which carries the information about entrance and exit channels.
\end{abstract}

The time independent mean field theory of collisions (TIMF) [1] has been tested successfully for solvable cases of 3 and 4 particles [2] and has been applied to various nuclear and atomic reactions [3]. It is then natural to go beyond the mean field approach by introducing particlehole $(p h)$ correlations in analogy to the case of bound states. In this letter we outline how this goal can be reached by a generalized random phase approximation. Our approach can be viewed as an alternative of more traditional inclusions of correlations into scattering theories, such as the antisymmetrization of cluster structures [4] and fluctuations into time dependent Hartree-Fock [5], for instance.

We start from a time independent functional [1] which calculates the Green function $D(z)$ between incoming and outgoing channel waves $\chi, \chi^{\prime}$. The variation of

$$
F\left(\Psi^{\prime}, \Psi\right)=<\chi^{\prime}\left|\Psi><\Psi^{\prime}\right| \chi>/<\Psi^{\prime}|(z-H)| \Psi>,
$$

with $\operatorname{Im} z>0$ gives, in appropriate normalization,

$$
\begin{gathered}
(z-H)|\Psi>=| \chi>, \quad<\Psi^{\prime}\left|(z-H)=<\chi^{\prime}\right|, \\
D(z)=<\chi^{\prime}\left|(z-H)^{-1}\right| \chi>=<\chi^{\prime}\left|\Psi>=<\Psi^{\prime}\right| \chi>,
\end{gathered}
$$

with $\Psi, \Psi^{\prime}$ as stationary (saddle!) points of (1). For simplicity we assume that $\chi$ and $\chi^{\prime}$ are Slater determinants made of $N$ square integrable orbitals $\chi_{i}$ and $\chi_{i}^{\prime}$, respectively. $H$ is the standard full Hamiltonian of $N$ particles. In the same way one may obtain the $T$-matrix by multiplying $\chi, \chi^{\prime}$ by prior and post potentials $V, V^{\prime}$ in the above equations. While one normally calculates $D$ as function of (complex) energy $z$, we now reverse the strategy: We rewrite (2) with (3) as

$$
\begin{aligned}
& (z-H)|\Psi>=\Lambda| \chi><\chi^{\prime} \mid \Psi>, \\
& <\Psi^{\prime}\left|(z-H)=\Lambda<\Psi^{\prime}\right| \chi><\chi^{\prime} \mid,
\end{aligned}
$$

which we interpret as right and left eigenvalue equations of the non-hermitean Hamiltonian

$$
H^{\prime}=H+\Lambda\left|\chi><\chi^{\prime}\right| ; \quad \Lambda=1 / D \text { complex },
$$

with eigenvalues $z=z(\Lambda)$. Choosing fixed values of $\Lambda$ in (4) and (5) preserves the equivalence of (2) and (4), since Eqs.(4) are invariant under normalization of $\Psi, \Psi^{\prime}$ and their solutions are stationary points of the functional (1) which also is invariant under normalization of $\Psi, \Psi^{\prime}$.

The discrete eigenvalues of $H^{\prime}$ are solutions of the obvious quantization condition

$$
\Lambda^{-1}=<\chi^{\prime}\left|(z-H)^{-1}\right| \chi>,
$$

which holds for both right and left eigenstates according to Eqs.(4). It is clear that any real $E$ of the discrete spectrum of $H$ generates a complex $z$ close to $E$, at least whenever $\Lambda$ is small. Conversely, there could be discrete eigenvalues $z$ of $H^{\prime}$ which are not in the vicinity of discrete eigenvalues of $H$. As long as $\Lambda$ is the reciprocal of a physical amplitude $D$, an additional, discrete, complex eigenvalue emerges, close to the physical scattering energy $E$. The continuum of $H^{\prime}$ is the same as that of $H$ because of the compactness [6] of $\left|\chi><\chi^{\prime}\right|$.

Square integrable left and right eigenstates of $H^{\prime}$ can be found from the Rayleigh-Ritz-like functional

$$
F^{\prime}\left(\Psi^{\prime}, \Psi\right)=<\Psi^{\prime}\left|\left(z-H^{\prime}\right)\right| \Psi>,
$$

where $z$ appears as a norm and phase control Lagrange multiplier, actually equal to the desired eigenvalue of $H^{\prime}$, and the trial functions $\Psi, \Psi^{\prime}$ are square integrable as long as $\operatorname{Im} z \neq 0$. Indeed, the variational equations of (7) induce the right and left eigenvalue equations (4). It should be stressed here that, from the point of view of 
the variational principle for $F^{\prime}$, one looks for $z$ when $\Lambda$ is given, while from the point of view of $F$, one looks for $\Lambda$ when $z$ is given.

In this paper we investigate solutions of (4) where Re $z$ can be interpreted as an energy of the continuum of $H$, with $\operatorname{Im} z>0$, and we consider $z$ as a function of $\Lambda$. In other words, we seek an energy $z$ for which the Green function $D(z)$ is equal to a given value $\Lambda^{-1}$. Our way of solving (6) for $z$ consists in diagonalizing $H^{\prime}$ via suitable approximations of $\Psi, \Psi^{\prime}$ in the functional (7). This involves first a Hartree-Fock approximation which induces a zero order answer $z_{0}(\Lambda)$, then possibly a Tamm-Dancoff approach (TDA) to the spectrum of $H^{\prime}$ and finally random phase (RPA), second order corrections $\Delta z(\Lambda)$, with the final result $z_{2}(\Lambda)=z_{0}(\Lambda)+\Delta z(\Lambda)$. First order corrections are automatically cancelled by the Hartree-Fock method (generalized Brillouin theorem).

The Hartree-Fock approximation to the diagonalization of $H^{\prime}$ consists in restricting $\Psi, \Psi^{\prime}$ to just Slater determinants $\Phi, \Phi^{\prime}$, made of orbitals $\varphi_{i}, \varphi_{i}^{\prime}, i=1,2, \ldots N$. To simplify the corresponding variational equations, we redefine $\chi_{i}, \chi_{i}^{\prime}$ by the conditions

$$
<\varphi_{i}^{\prime}\left|\chi_{j}>=0=<\chi_{i}^{\prime}\right| \varphi_{j}>\text { for } i \neq j
$$

This just means a linear rearrangement of the orbitals $\chi_{i}, \chi_{i}^{\prime}$, leaving the determinants $\chi, \chi^{\prime}$ invariant. Then the term $\Lambda<\Psi^{\prime}\left|\chi><\chi^{\prime}\right| \Psi>$ is a product of overlaps $<\varphi_{i}^{\prime}\left|\chi_{i}><\chi_{j}^{\prime}\right| \varphi_{j}>$ and the Hartree-Fock equations read

$$
\begin{gathered}
\left(\eta_{i}-h\right)\left|\varphi_{i}>=\lambda_{i}\right| \chi_{i}><\chi_{i}^{\prime} \mid \varphi_{i}> \\
<\varphi_{i}^{\prime}\left|\left(\eta_{i}-h\right)=\lambda_{i}^{\prime}<\varphi_{i}^{\prime}\right| \chi_{i}><\chi_{i}^{\prime} \mid
\end{gathered}
$$

$\lambda_{i}=\lambda_{i}^{\prime}$ reciprocal of the single-particle Green function,

$$
\lambda_{i}=\lambda_{i}^{\prime}=\left(<\chi_{i}^{\prime}\left|\left(\eta_{i}-h\right)^{-1}\right| \chi_{i}>\right)^{-1} .
$$

The operator $h=t+U$ contains a self consistent mean field $U$, similar to the standard Hartree-Fock potential, and the propagation energies $\eta_{i}$ are also given by self consistent formulae similar to those defining Hartree-Fock self energies. From (9), (10) we immediately read off the Hartree-Fock Hamiltonian corresponding to $H^{\prime}$,

$$
h^{\prime}=h+\sum_{j=1}^{N} \lambda_{j}\left|\chi_{j}><\chi_{j}^{\prime}\right|
$$

bearing in mind (8). Standard manipulations of (9), together with (8), show that $\varphi_{i}, \varphi_{i}^{\prime}$ are biorthogonal. Finally one can use normalizations such that

$$
\begin{aligned}
& <\varphi_{i}^{\prime}\left|\varphi_{j}>=\delta_{i j} ;<\varphi_{i}^{\prime}\right| \chi_{j}>=<\chi_{i}^{\prime} \mid \varphi_{j}>=\gamma_{i} \delta_{i j}, \\
& \gamma_{i}=\frac{<\chi_{i}^{\prime}\left|\left(\eta_{i}-h\right)^{-1}\right| \chi_{i}>}{\sqrt{<\chi_{i}^{\prime}\left|\left(\eta_{i}-h\right)^{-2}\right| \chi_{i}>}}
\end{aligned}
$$

since there are still infinitely many ways to adjust the phases and norms of $\chi_{i}, \chi_{i}^{\prime}$ in such a way that $\chi, \chi^{\prime}$ remain unchanged. We then extend the diagonalization of $h^{\prime}$ beyond the first $N$ right and left eigenstates, and obtain an infinite, biorthonormal set of orbitals $\varphi_{\alpha}, \varphi_{\beta}^{\prime}$, presumably complete. This set most likely includes continuum states, to be normalized accordingly, hence

$$
\begin{gathered}
<\varphi_{\beta}^{\prime} \mid \varphi_{\alpha}>=\delta_{\beta \alpha} \text { or } \delta\left(\eta_{\beta}-\eta_{\alpha}\right) \\
\left(\eta_{\alpha}-h^{\prime}\right)\left|\varphi_{\alpha}>=0,<\varphi_{\beta}^{\prime}\right|\left(\eta_{\beta}-h^{\prime}\right)=0 .
\end{gathered}
$$

The equivalence of inversion and diagonalization on the many-particle level, based on the functionals $F\left(\Psi^{\prime}, \Psi\right)$ and $F^{\prime}\left(\Psi^{\prime}, \Psi\right)$, is reflected on the single-particle level: The homogeneous equations (9) for $h^{\prime}$ are strictly equivalent to the inhomogeneous TIMF equations,

$$
\left(\eta_{i}-h\right)\left|\varphi_{i}>=\right| \chi_{i}>,<\varphi_{i}^{\prime}\left|\left(\eta_{i}-h\right)=<\chi_{i}^{\prime}\right|
$$

obtained from the functional $F\left(\Phi^{\prime}, \Phi\right)$ with $\Phi, \Phi^{\prime}$ as Slater determinants. In particular the eigenvalues of $h^{\prime}(\Lambda=0)=h$, Eq.(15), coincide with the poles of $D$, where $\Lambda=0$, when calculated from Eqs.(16).

To improve the mean field approach by $p h$ correlations, we introduce creation and annihilation operators $a_{\alpha}^{\dagger}, a_{\alpha}, \alpha=1,2 \ldots \infty$ for orbitals $\varphi_{\alpha} ; a_{\beta}^{\prime \dagger}, a_{\beta}^{\prime}, \beta=$ $1,2 \ldots \infty$ are the corresponding operators for $\varphi_{\beta}^{\prime}$. Finally a set of operators $J_{i}^{\dagger}, J_{i}, i=1,2 \ldots N$ is introduced for orbitals $\chi_{i}$, and a similar set $J_{i}^{\prime \dagger}, J_{i}^{\prime}$ for orbitals $\chi_{i}^{\prime}$. A second quantization representation of $H^{\prime}$ is then

$$
\begin{aligned}
H^{\prime}= & \sum_{\alpha \beta}<\varphi_{\alpha}^{\prime}|t| \varphi_{\beta}>a_{\alpha}^{\dagger} a_{\beta}^{\prime} \\
& +\frac{1}{4} \sum_{\alpha \beta \gamma \delta}<\varphi_{\alpha}^{\prime} \varphi_{\gamma}^{\prime}|v| \varphi_{\beta} \varphi_{\delta}>a_{\alpha}^{\dagger} a_{\gamma}^{\dagger} a_{\delta}^{\prime} a_{\beta}^{\prime} \\
& +\Lambda J_{1}^{\dagger} J_{2}^{\dagger} \cdots J_{N}^{\dagger} J_{N}^{\prime} \cdots J_{2}^{\prime} J_{1}^{\prime} .
\end{aligned}
$$

In (17) one set only of operator pairs $a_{\alpha}^{\dagger}, a_{\beta}^{\prime}$ appears. It anticommutes canonically according to (14). With $\mid 0>$ as fermion vacuum we have

$$
\left|\Phi>=\prod_{i=1}^{N} a_{i}^{\dagger}\right| 0>,<\Phi^{\prime}|=<0| \prod_{i=1}^{N} a_{i}^{\prime}
$$

as right and left quasi-particle vacua. Hence a complete set of $p h$ operators is given by

$$
B_{m i}^{\dagger}=a_{m}^{\dagger} a_{i}^{\prime}, B_{m i}^{\prime}=a_{m}^{\prime} a_{i}^{\dagger}
$$

for $i=1,2 \ldots N, m=N+1, \ldots, \infty$, and the ansatz for boson operators on the RPA level is

$$
Q_{\nu}=\sum_{m i}\left(X_{m i}^{\nu} B_{m i}^{\dagger}-Y_{m i}^{\nu} B_{m i}^{\prime}\right)
$$

If one sets $Y_{m i}^{\nu}=0$ a priori, one falls back to the TDA. 
While Wick's theorem is available to calculate the matrix elements of kinetic energy $T$ and two-particle interaction $V$, the matrix elements of $\Lambda\left|\chi><\chi^{\prime}\right|$ are less familiar. They are simplified, however, in the biorthogonal representation (12), and read,

$$
\begin{aligned}
<\Phi^{\prime}\left|a_{i}^{\dagger} a_{m}^{\prime}\right| \chi> & =<\Phi^{\prime}\left|\chi><\varphi_{m}^{\prime}\right| \chi_{i}>/<\varphi_{i}^{\prime} \mid \chi_{i}>, \\
<\chi^{\prime}\left|a_{m}^{\dagger} a_{i}^{\prime}\right| \Phi> & =<\chi^{\prime}\left|\Phi><\chi_{i}^{\prime}\right| \varphi_{m}>/<\chi_{i}^{\prime} \mid \varphi_{i}>, \\
<\Phi^{\prime}\left|a_{i}^{\dagger} a_{j}^{\dagger} a_{n}^{\prime} a_{m}^{\prime}\right| \chi>= & \frac{<\Phi^{\prime}\left|\chi><\varphi_{m}^{\prime} \varphi_{n}^{\prime}\right| \chi_{i} \chi_{j}>}{<\varphi_{i}^{\prime}\left|\chi_{i}><\varphi_{j}^{\prime}\right| \chi_{j}>}, \\
<\chi^{\prime}\left|a_{m}^{\dagger} a_{n}^{\dagger} a_{j}^{\prime} a_{i}^{\prime}\right| \Phi>= & \frac{<\chi^{\prime}\left|\Phi><\chi_{i}^{\prime} \chi_{j}^{\prime}\right| \varphi_{m} \varphi_{n}>}{<\chi_{i}^{\prime}\left|\varphi_{i}><\chi_{j}^{\prime}\right| \varphi_{j}>} .
\end{aligned}
$$

Let $M$ be the number of $p h$ pairs ( $m i)$ retained in a practical calculation. The $2 M$ solutions of the RPA equations

$$
\begin{aligned}
& <\Phi^{\prime}\left|\left[\left[H^{\prime}, Q_{\nu}\right], B_{n j}^{\dagger}\right]\right| \Phi>=E_{\nu}^{*}<\Phi^{\prime}\left|\left[Q_{\nu}, B_{n j}^{\dagger}\right]\right| \Phi> \\
& <\Phi^{\prime}\left|\left[\left[H^{\prime}, Q_{\nu}\right], B_{n j}^{\prime}\right]\right| \Phi>=E_{\nu}^{*}<\Phi^{\prime}\left|\left[Q_{\nu}, B_{n j}^{\prime}\right]\right| \Phi>
\end{aligned}
$$

then split into 2 families of $M$ solutions. For the first family, $E_{\nu}^{*}$ denotes the "excitation" energy which separates the RPA-correlated eigenstate $|\Psi\rangle$ based on $|\Phi\rangle$ from another eigenstate of $H^{\prime}$ defined by the RPA ansatz $\left|\Psi_{\nu}>=Q_{\nu}\right| \Psi>$. In the second family, for which we will use a label $\bar{\nu},-E_{\nu}^{*}$ is the energy difference between $<\Psi_{\bar{\nu}}^{\prime}\left|=<\Psi^{\prime}\right| Q_{\bar{\nu}}$ and the correlated state $<\Psi^{\prime} \mid$ based on $<\Phi^{\prime} \mid$. The solutions $\nu$ of the first family can be told from those $\bar{\nu}$ of the second family by selecting as $E_{\nu}^{*}$ those RPA eigenvalues close to the eigenvalues of the TDA, which provides only $M$ solutions. A straightforward, slightly tedious calculation, gives for (22)

$$
\begin{aligned}
& \sum_{m i}\left(C_{n j, m i} X_{m i}^{\nu}+A_{m i, n j} Y_{m i}^{\nu}\right)=-E_{\nu}^{*} Y_{n j}^{\nu} \\
& \sum_{m i}\left(A_{n j, m i} X_{m i}^{\nu}+B_{n j, m i} Y_{m i}^{\nu}\right)=+E_{\nu}^{*} X_{n j}^{\nu}
\end{aligned}
$$

with

$$
\begin{aligned}
& C_{n j, m i}=-<\varphi_{i}^{\prime} \varphi_{j}^{\prime}|v| \varphi_{m} \varphi_{n}>-\bar{\Lambda} \frac{<\chi_{i}^{\prime} \chi_{j}^{\prime} \mid \varphi_{m} \varphi_{n}>}{<\chi_{i}^{\prime}\left|\varphi_{i}><\chi_{j}^{\prime}\right| \varphi_{j}>}, \\
& B_{n j, m i}=-<\varphi_{m}^{\prime} \varphi_{n}^{\prime}|v| \varphi_{i} \varphi_{j}>-\bar{\Lambda} \frac{<\varphi_{m}^{\prime} \varphi_{n}^{\prime} \mid \chi_{i} \chi_{j}>}{<\varphi_{i}^{\prime}\left|\chi_{i}><\varphi_{j}^{\prime}\right| \chi_{j}>},
\end{aligned}
$$

$$
\begin{aligned}
A_{n j, m i}= & \delta_{m n} \delta_{i j}\left(\eta_{m}-\eta_{i}\right)-<\varphi_{n}^{\prime} \varphi_{i}^{\prime}|v| \varphi_{m} \varphi_{j}> \\
& +\bar{\Lambda}\left\{\frac{<\chi_{i}^{\prime}\left|\varphi_{m}><\varphi_{n}^{\prime}\right| \chi_{j}>}{<\chi_{i}^{\prime}\left|\varphi_{i}><\varphi_{j}^{\prime}\right| \chi_{j}>}\right. \\
& \left.-\delta_{i j} \sum_{l} \frac{<\varphi_{n}^{\prime}\left|\chi_{l}><\chi_{l}^{\prime}\right| \varphi_{m}>}{<\chi_{l}^{\prime}\left|\varphi_{l}><\varphi_{l}^{\prime}\right| \chi_{l}>}\right\},
\end{aligned}
$$

using $\bar{\Lambda}=\Lambda<\Phi^{\prime}\left|\chi><\chi^{\prime}\right| \Phi>$ and the relation

$$
\lambda_{i}=\lambda_{i}^{\prime}=\bar{\Lambda} /<\varphi_{i}^{\prime}\left|\chi_{i}><\chi_{i}^{\prime}\right| \varphi_{i}>
$$

Eqs.(23) show less symmetry than the usual RPA equations [7], as expected from the non-hermitean $H^{\prime}$ as compared to $H$. However, $B$ and $C$ are symmetric under exchange of the index pairs $(m i)$ and $(n j)$, the block matrix

$$
S=\left(\begin{array}{cc}
C & \tilde{A} \\
A & B
\end{array}\right)
$$

is symmetric and Eqs.(23) read

$$
S\left(\begin{array}{l}
X^{\nu} \\
Y^{\nu}
\end{array}\right)=E_{\nu}^{*} \mathcal{M}\left(\begin{array}{l}
X^{\nu} \\
Y^{\nu}
\end{array}\right), \text { with } \mathcal{M}=\left(\begin{array}{cc}
0 & -1 \\
1 & 0
\end{array}\right) .
$$

It is then easy to show that the "symplectic diagonalization" problem (29) is equivalent to diagonalizing the antisymmetric matrix

$$
\mathcal{A}=S^{-1 / 2} \mathcal{M} S^{-1 / 2},
$$

namely

$$
\mathcal{A} S^{1 / 2}\left(\begin{array}{l}
X^{\nu} \\
Y^{\nu}
\end{array}\right)=E_{\nu}^{*-1} S^{1 / 2}\left(\begin{array}{l}
X^{\nu} \\
Y^{\nu}
\end{array}\right)
$$

An elementary derivation of (31) from (29) involves the definition of any symmetric square root of $S$ and its inversion, and the assumption that $E_{\nu}^{*} \neq 0$. While translational and rotational symmetry of $H$ give rise to null eigenvalues in usual $R P A$, the $\left|\chi><\chi^{\prime}\right|$-term in $H^{\prime}$ destroys these symmetries, hence eigenvalues $E_{\nu}^{*}=0$ are usually not to be expected. Special proofs are available when an exceptional incident occurs.

The fact that $\mathcal{A}$ is antisymmetric proves that the spectrum splits into two families of opposite eigenvalues, as announced. Consider now the $M$ eigenvalues $E_{0 \nu}^{*}$, coming from the diagonalization of $A$, and define the auxiliary "perturbative RPA" matrix

$$
S_{\epsilon}=\left(\begin{array}{cc}
\epsilon C & \tilde{A} \\
A & \epsilon B
\end{array}\right), \quad 0 \leq \epsilon \leq 1
$$

with corresponding $\mathcal{A}_{\epsilon}$. It is clear that, as $\epsilon \rightarrow 0$, half of the eigenvalues of the auxiliary problem (32) converge towards $E_{0 \nu}^{*}$, while the other $M$ eigenvalues tend to $-E_{0 \nu}^{*}$. By continuity when $\epsilon \rightarrow 1$, this makes it easy to separate the eigenvalues of (29) into the two announced families, both being complex due to the nonhermiticity of $H^{\prime}$. With the first family, the usual bosonic interpretation of the RPA-like Hamiltonian finally provides the correction

$$
\Delta z(\Lambda)=\frac{1}{2}\left(\sum_{\nu=1}^{M} E_{\nu}^{*}-\operatorname{Tr} A\right)
$$

A function inversion of the final result $z_{2}(\Lambda)=z_{0}(\Lambda)+$ $\Delta z(\Lambda)$, generating a function $\Lambda\left(z_{2}\right)$, then provides the 
improvement on the Green function, beyond the mean field, by inclusion of $p h$ correlations.

In conclusion, the key element of our study is the equivalence of the following two problems:

1.) Calculate the Green function $D(z)$ at some fixed (complex) energy $z$ by operator inversion of the exact Hamiltonian $H$. One solves an inhomogeneous problem with channel wave functions $\chi, \chi^{\prime}$ as inhomogeneity.

2.) Diagonalize a Hamiltonian $H^{\prime}$ which differs from $H$ by the operator $\Lambda\left|\chi><\chi^{\prime}\right|$, comprising the reaction boundary conditions. In this homogeneous approach, one calculates the (complex) eigenvalues $z$ as function of the parameter $\Lambda=1 / D$ and obtains the desired Green function by inversion of the function $z(\Lambda)$.

Thus operator inversion is replaced by operator diagonalization followed by function inversion. While problems 1.) and 2.) can equally well be solved in the mean field approximation, we use 2.) to incorporate correlations in the wave function in terms of $p h$ bosons. Although the resulting boson Hamiltonian is less symmetric than in standard (hermitean) RPA, the remaining symmetry is sufficient to diagonalize the (non-hermitean) boson Hamiltonian by canonical, but non-unitary transformation and to calculate $z(\Lambda)$ in this boson approximation.

We tested numerically the above method for a onedimensional, separable, two-body Hamiltonian of relative motion

$<q^{\prime} Q^{\prime}|\mathcal{H}| q Q>=$

$\delta\left(Q-Q^{\prime}\right)\left[q^{2} \delta\left(q-q^{\prime}\right)-\lambda \nu^{2} q q^{\prime} \exp \left(-\nu^{2} q^{2}\right) \exp \left(-\nu^{\prime 2} q^{\prime 2}\right)\right]$,

with $q, Q$ the relative and total momentum, which gives an obvious solution [3] for the exact amplitude $D(z)$ of (3). Our TIMF and RPA numerics, however, used the single-particle representation which becomes mandatory for antisymmetrized, many-particle calculations. The channel waves $\chi, \chi^{\prime}$ were taken as boosted Gaussian functions. Mass and potential parameters as well as boost and width of $\chi, \chi^{\prime}$ were chosen to be typical for nuclear physics problems; for technical details we refer to [8]. The RPA approximation of $D(z)$, based on (23) and (33), indeed improved the TIMF results (see Fig.1) obtained by solving (16).

One of us (A. W.) wants to thank the Japan Society for Promotion of Science and the Deutsche Akademische Austauschdienst for financial support during his stay in Japan. He is also very grateful for the hospitality found at the Yukawa Institute for Theoretical Physics in Kyoto.

* Permanent Address: Institut für Theoretische Physik, Universität Münster, 48149 Münster, Germany.
[1] B.G. Giraud, M.A. Nagarajan, and I.J. Thompson, Ann.Phys.(N.Y.) 152, 475 (1984).

[2] Y. Abe and B.G. Giraud, Nucl.Phys. A440, 311 (1985); J. Lemm, A. Weiguny, and B.G. Giraud, Z.Phys. A336, 179 (1990).

[3] B.G. Giraud, M.A. Nagarajan, and C.J. Noble, Phys.Rev.A 34, 1034(1986); B.G. Giraud and M.A. Nagarajan, Ann.Phys.(N.Y.) 212, 260(1991); B.G. Giraud, Y. Hahn, F. Mekideche, and J. Pascale, Z.Phys. D27, 295(1993).

[4] Y.C. Tang: Microscopic Description of the Nuclear Cluster Theory, Lecture Notes in Physics, Vol. 145 (Springer, New York, 1981).

[5] R. Balian and M. Vénéroni, Ann.Phys.(N.Y.) 195 (1989), 324.

[6] M. Reed and B. Simon: Methods of Modern Mathematical Physics. I: Functional Analysis (Academic Press, New York, 1972).

[7] D.J. Thouless, The Quantum Mechanics of Many Body Systems (Academic Press, New York, 1961).

[8] J.C. Lemm, doctoral dissertation, Münster University, 1993.
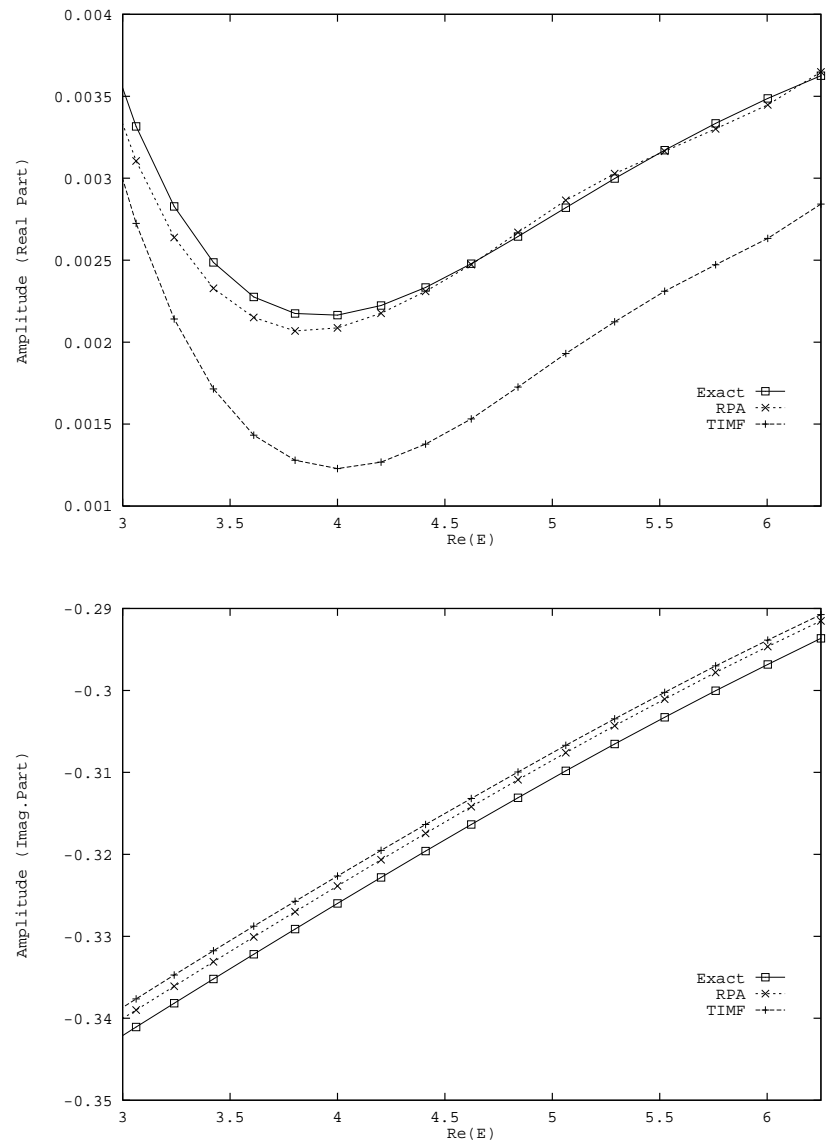

FIG. 1. Real and imaginary part of the exact, TIMFand RPA-amplitudes vs. Re $E$, at $\operatorname{Im} E=2.0$ for a solvable two-body model with gaussian interaction. 